identified at least two other patients who probably had acquired HBV infection from one of these surgeons. None of the surgeons had a history of reported needlestick injuries, none practiced double gloving routinely, and all were assessed as technically competent by their colleagues. Two of the surgeons had received HBV vaccination; however, they most likely acquired their infections prior to vaccination.

In a 1993 revision of the United Kingdom guideline on $\mathrm{HBV}$, all healthcare workers at risk for HBV infection must be vaccinated, and their immune response must be documented. Healthcare workers who perform procedures involving a risk of exposure, defined according to guidelines, and who do not have an antibody titer HBsAg of at least $10 \mathrm{mIU} / \mathrm{mL}$ after vaccination, are investigated further. Those in whom HBeAg is detected are not allowed to perform procedures involving a risk of exposure. Carriers in whom serum $\mathrm{HBeAg}$ is not detectable may perform such procedures, unless their participation is shown to be associated with the transmission of HBV.

They receive advice about preventing transmission and career counseling, but are in the unenviable position of having to weigh the prospect of future restrictions of practice against the desire to continue in their chosen career. All of the four surgeons in this investigation were given career counseling-all remain in medicine, three in work that includes contact with patients and the performance of invasive procedures, but not in procedures involving a risk of exposure to the virus. One surgeon continues to work in the same department but in a different capacity. One surgeon had considerable difficulty before the offer of alternative employment was made, as required by the current guideline.

The introduction of the guidelines in the United Kingdom may have contributed to the detection of these four cases by raising professional awareness about the possibility of transmission of HBV from surgeons to patients and by instituting the prospective restriction of practice of HBeAg-positive surgeons.

Three of the four cases were identified after the introduction of the guidelines. The investigators note that a better understanding of the markers that predict transmission would benefit infected surgeons, as well as those responsible for public policy development.

FROM: Heptonstall $\mathrm{J}$, the Incident Investigation Team. Transmission of hepatitis B to patients from four infection surgeons without hepatitis B e antigen. $N$ Engl J Med 1997;336:178-184.

\section{Meningococcal Infection Transmitted From Saliva}

Investigators from the Lambeth, Southwark, and Lewishan Health Authorities in London, England, recently reported a case of occupationally acquired conjunctival infection with Neisseria meningitidis following an incident involving continual exposure to saliva. The case involved a hospital security guard who developed hemorrhagic conjunctivitis 2 days after a man spit in his eye, with $N$ meningitidis being isolated from a conjunctival swab culture. $N$ meningitidis also was isolated from nasopharyngeal swabs from the man who spit in the security guard's eye and the man's household partner.

All three strains were found to be serogroup B, serotype 4 , and also were found to be sulfonamide resistant, yielding the phenotype B $4 \mathrm{nt} \mathrm{R}$. Macrorestriction profiles showed that all three isolates were genotypically closely related.

$N$ meningitidis is an uncommon causative organism of acute conjunctivitis. Until the introduction of antimicrobial therapy, endogenous meningococcal conjunctivitis was a known complication of systemic meningococcal disease. Systemic meningococcal disease from primary meningococcal conjunctivitis develops in approximately $18 \%$ of patients. This report illustrates how a meningococcus bearing the phenotype of a well-recognized invasive strain can cause serious infection within 48 hours.

FROM: Holdsworth G, Jackson H, Kaczmarski E. Meningococcal infection from saliva. Lancet 1996;348:1443. Letter.

Additional news items in this issue: Candida Endocarditis From Contaminated Aortic Valve, page 411; VRE Skin Colonization and Risk of Bacteremia, page 416. 